\title{
Advantages and Disadvantages of using Food Grate Stainless Steel Rods for Internal Fixation of Femoral Transverse Fractures in Rabbits (Review Study)
}

\author{
Humam H Nazht ${ }^{1 *}$, Ammar MH Almahmoud ${ }^{2}$, Raffal A Omar ${ }^{1}$, Zyad \\ Maher Manual' ${ }^{3}$, Zied Husain Dyab3 Rania Khedr Khaleefa ${ }^{3}$ and Sumaya \\ Mohammed Kareem ${ }^{3}$ \\ ${ }^{1}$ Surgery and obstetric department, College of veterinary medicine, University of Baghdad, \\ Iraq
}

${ }^{2}$ Surgery and obstetric department, College of veterinary medicine, Albasra university, Iraq ${ }^{3}$ Private clinic, Baghdad, Iraq

*Corresponding author: Humam H Nazht, Surgery and obstetric department, college of veterinary medicine, university of Baghdad, Iraq, Tel: 009647901784013; Email: Humam_Nazhat@yahoo.com

\section{Abstract}

The present study designed to focus on the advantages and disadvantages of using food grate stainless steel rods (FGSR) as internal fixation methods for induced transverse fractures in the mid shift of the femoral bones in rabbits.200 cases were collected from 2007 to 2020, all these cases were employed to induced transverse fractures in the mid shift of femoral bone, 100 of them used the rods for internal fixation, 80 of the cases used the rods for fixation the natural xen- bony implantation from sheep or calves, and the others 20 cases used the rods for internal fixation of the synthetics nano bony implantation.The physical, chemical, clinical and radiographic parameters were used for evaluation, the physical and chemical analysis showed that the rods not change during sterilization, implantation or when exposed to different types of ray besides the chemical constant is about the same level measurement of the medical intramedullary pins (IMP), while the clinical observation revealed that the rods can used strongly and successfully for fracture fixation and support the animal to bear the weight, the limb used for walking gradually after 24-48 hours p. 0 . with some cases shown the FGSR pulled from the bone, other suffer from infection with pus formation with lateral deviation of the stifle joint or re-fracture. While the radiological finding revealed that the FGSR insert and fix the fractures fragment and the bony implantation, the pins seemed stable and fit the intramedullary canal and fracture fragments, while some cases shows that the pin not insert properly inside the femoral bone with case of multible and comminuted fractures, other case shown the FGSR pass and penetrate the stifle joint. The conclusion is, there are many advantages with minor or very little disadvantages for using the FGSR as internal fixation of the induced transverse fractures in the mid shift of the femoral bones and for natural and synthetics bony implantation in rabbits.

Keywords: Food Grate Stainless Steel Rods (FGSR); Induced Transverse Fractures; Intramedullary Pinning (IMP); XenoBony Implantation; Synthetic Nano-Bony Implantation 


\section{Open Access Journal of Veterinary Science \& Research}

\section{Introduction}

Fractures can be defined as a medical condition in which there is damage in the continuity of the bone, can be results from high force impact or stress, or from a certain medical conditions that weaken the bones [1-3].

Generally, fractures can be fixed either by internal fixation with intramedullary pin, bone plate with screw, stainless steel wire, or by external fixation like plaster of pairs, plastic casts, mason meta, and modified Thomas splints and semi cast, or may be combination between two internal fixation methods or between internal and external fixation like pinning with circler stainless steel wire, bone plate with screw with external splint $[1,2]$.

Fixation by intramedullary pinning (IMP) has become the stander of treatment for long bone fractures, this technique is success and easy, commonly used, and less traumatic to ill animal $[4,5]$.

Nazht Humam H, et al. [6] conclude that using food grate stainless steel rods (FGSR) size $2.25-2.5 \mathrm{~mm}$ can be used strongly and successfully in internal fixation of induced transverse mid shift fractures of femoral bones and fixation of the natural and synthetics bony implantation in rabbits instead of the medical IMP and the results can be determined radiographically [7-11].

IMP are usually made of titanium and stainless steel, come in various lengths and diameters to fit most femur bones, with several advantages, including lower mechanical failure rates and improved biocompatibility $[12,13]$. And one of the significant advantages of IMP over other methods of fracture fixation is that IMP share the load with the bone, rather than entirely supporting the bone. Because of this, patients are able to use the extremity more quickly. IM implants don't really speed bone healing and they don't take the place of bone healing, but to hold the fracture in proper alignment while the normal healing process takes place. Some IM rods are designed to have sufficient strength to carry the load of the body, and that is why people can often place weight on the extremity sooner than if you had to wait for full healing of the bone [13].

Frik EJ, et al. [14], refer to the advantages of using IMR for internal fixation in that they easy to use with low cost, Less of bone exposed comparing with other methods (bone plate), with minimal amount of tissue damage and distraction, and do not need to support it with the external fixation, and do not lead to deformity of loss the function of the affected limbs or any joint stiffness, and according to the advantages above it have highly union rate of the femur about $97 \%[4,5]$. while the disadvantages of using the IMP for the internal fixation may not lead to immobilization, not stopped the circulation movement, lead to bone marrow destruction and the inner blood circulation and may lead to infection due to interance of microorganism, may lead to osteomylitis, may lead to loss in the pins, less stable fixation, slower return to function, secondary bone union, more involved after care $[14,15]$.

The aim of this study is to evaluate the advantages and disadvantages of FGSR for internal fixation of the induced fracture in the femoral bone in rabbits from reviewing the cases from January 2007 until March 2020 in veterinary surgery and obstetric department/college of veterinary medicine/university of Baghdad

\section{Materials and Methods}

The data were collected from 200 practical from January 2007 - March 2020 as following, 100 cases employed for bone grafting / implantation, 80 case used to induced two transverse fractures in the mid shift of the femoral bone to remove about $1 \mathrm{~cm}$ of the bone and replaced by the same size and length of natural Xeno bony implantation from sheep ribs, or radial bone of calves, they fixed by FGSR, the animals divided to control and two treatment group which exposed to different dosage of low level laser therapy ( LLLT) and electromagnetic field therapy. And the remaining 20 cases were employed to remove $1 \mathrm{~cm}$ of femoral bone and replaced by synthetic bony nano material (which prepared in the physical laboratory of college of science A-lmustanseria University /Iraq) and follow for normal healing processing. 100 cases induced transverse fracture in the mid shift of femoral bone of rabbits and fixed by FGSR

(Figure 1) and divided to control and treatment group which exposed to LLLT and followed for healing processing.

\section{Evaluation of the Experimental Study}

All the cases evaluated by the following parameters [6-11].

1. Physical and chemical analysis to the FGSR

2. Daily clinical observation

3. Weekly radiographic examination.

\section{Food Grade Stainless Steel Rods (FGSR) Preparation}

Cutting FGSR (each rod $90-100 \mathrm{~cm}$ length and 2.25$2.5 \mathrm{~mm}$ diameter) to $10-11$ pieces $7.5-8.5 \mathrm{~cm}$ length, after sterilization (Nazht, 2019), the rods used for internal fixation under general anesthesia [16], with highly aseptic technique (Figure 2). The physical and chemical analysis down to both the FGSR and medical intramedullary pins (IMP) in the AlNahreen University. 

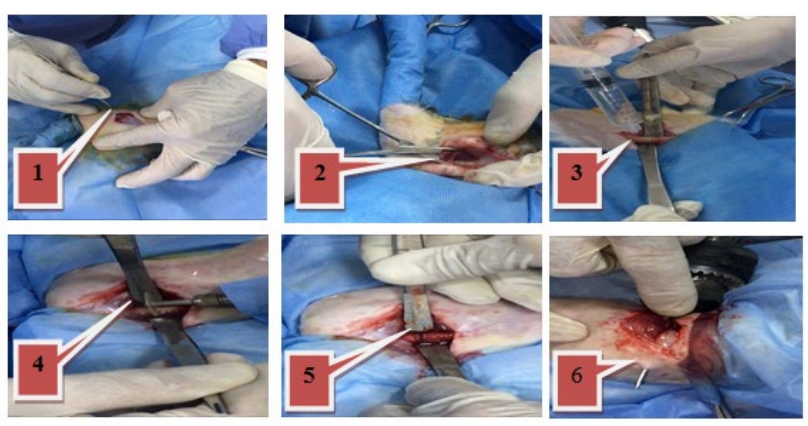

Figure 1: Surgical operation to implan FGSR.1.Site of incision, 2. Dissect the underlying soft tissues, 3. Exposed the femoral bone, 4 . Induced transverse fracture in the mid shift of the femoral bone, 5.Creat complete transverse fracture, 6. Inset the FGSR in the bone marrow canal.
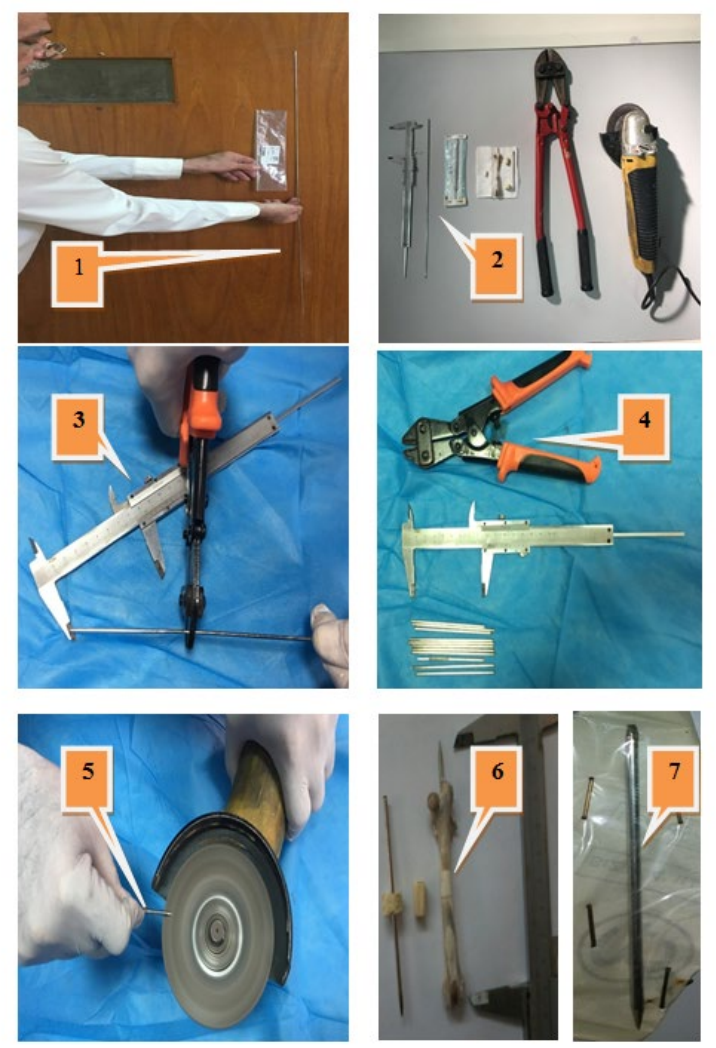

Figure 2: Steps of FGSR preparation, 1. Lenth of FGSR compare with the medical (IMP), 2. Tools requirement for FGSR preparation, 3. Measure and cutting the FGSR, 4. 10-11 piece of FGSR, 5. Sharpining the end of the FGSR, 6. FGSR used for fixation of natural and synthetic bony implantation, 7. Strilaization of the prepared FGSR.

\section{Results}

\section{The Results of the Physical and Chemical Analysis [6-11].}

- The physical and chemical analysis have the same or near the same measurement level in both the FGSR and the IMP, (piece of each rods send to physical laboratory in Al-Nahreen university for lab. analysis )

- The FGSR not change during different types of sterilization.

- The FGSR not change when exposed to different types of radiation.

- The FGSR not change when exposed to electromagnetic field.

- The FGSR when pulled out of the body during healing processing not change.

- The FGSR give positive results with hardness test and give strength and support to the fracture limb.

\section{The Results of the Clinical Observation [6-11].}

\section{$>$ Advantages:}

- The daily clinical observation revealed inflammatory signs, loss appetite during several hours P.O. which disappear within two days P.O.

- The experimental animals begin to use the limb in walking gradually from the first week until the end of the healing processing period.

- The FGSR support the fracture limb and the animals can bear the body weight during the walking, running, and jumping during fracture healing processing.

- There was no body rejection or complication during FGSR implantation.

- FGSR can be easy removed after fracture healing

$>$ Disadvantages:

- 3 cases suffering from infection and pus formation from the site of FGSR insertion, because the pin moved freely outside the body.

- 3 cases showed that the FGSR pulled outside the skin with limb deviation when walking and support body weight

- 10 cases show lateral deviation at the stifle joint during walking within two month p. o. then eight of them return normally and two remain with lateral deviation at the stifle joint until the end of the experimental period.

- 8 case die after surgical operation 4 of them at the first 24 hours p. o. and

- 2 cases of them within the first month p. o. and the last 2 cases die at the end of the one and half month p. 0 .

- Some case suffering from stitch abscess, wound dehisces.

- Little case showed great swelling at the surgical sites with pus formation at the surgical operation. 


\section{Open Access Journal of Veterinary Science \& Research}

\section{The Results of the Radiological Finding}

$>$ Advantages:

- Well fragment stability with good healing processing which evaluated radiographically (Figure 3.1).

- The pin can be removed from the fracture femoral bone after the radiological union at the end of the six week with clear signs of the hard callus formation and disappears of the fracture line (Figure 3.2).

- Type of fracture healing by secondary bone repair by endochondral ossification .with new hard callus formation continued by remodeling phase

$>$ Disadvantages:

- Excessive amount of the new bone formation around the fracture site

- Multiple bone fractures especially at the distal fragment or a site of fracture (Figure 4.1 and 4.2).

- The FGSR penetrate distally to the stifle joint (Figure 4.3.)

- The pin may not insert in the distal fragments or it may be out (Figure 4.4).

- None or mal union may be occurs during healing fractures processing.

- The FGSR fill bony canal and interfere with bone marrow and circulation.

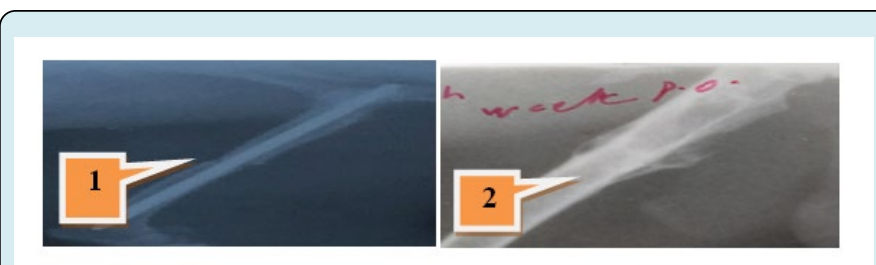

Figure 3: The advantages of using FGSR, Radiographic finding of internal fixation of the FGSR. 1. Proper fixation of the complete induced transverse femoral mid shift fracture first week p. o. 2. Remove the FGSR after fracture healing.
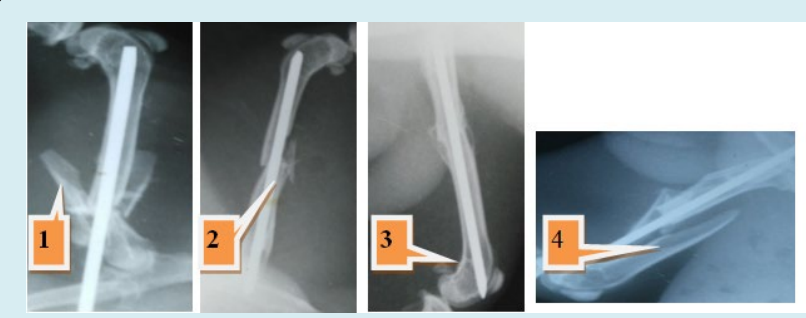

Figure 4: The disadvantages of using FGSR, 1. Multible fractures in the proximal fragment of femoral bone, 2. Chip fracture around the fracture site, 3 . The FGSR penetrate the distal end of the femoral bone in the stifle joint, 4. The FGSR not insert properly in the distal fragment of the femoral bone.

\section{Discussion}

\section{The Physical and Chemical Analysis}

\section{$>$ Advantages:}

FGSR and the medical IMP are similar for that both not change during sterilization, or when exposed to different types of rays that agree with [8], that using food grate stainless steel rods (FGSR) size $2.25-2.5 \mathrm{~mm}$ can be used strongly and successfully in internal fixation of induced transverse mid shift fractures of femoral bones in rabbits instead of the medical IMP, and both have the same physical and chemical properties, the FGSR made of stainless steel which lead to proper mechanical stability with no body rejection as mentioned by $[8,12,13]$ that Intramedullary nails are usually made of titanium or stainless steel come in various lengths and diameters to fit most femur bones and has several advantages, including lower mechanical failure rates and improved biocompatibility. Share the load with the bone

IM rods supporting the bone fragments and because of this, patients are able to use the extremity more quickly [6$10,11]$.

\section{$>$ Disadvantages:}

These types of implants don't really speed bone healing and they don't take the place of bone healing. Any metal implant used to stabilize a fracture is designed to hold the fracture in proper alignment while the normal healing process takes place [12,13].

While bone healing may not speed up by using the FGSR, they designed to have sufficient strength to carry the load of the body, and the patient place weight on the extremity sooner after implantation $[8,13]$.

\section{Clinical Observation}

\section{$>$ Advantages}

FGSR which used as Intramedellary pinning in femoral fracture fixation is popular easy to use, gives good stability and fixation and this is exactly what mentioned from others workers like $[4,5]$ they declare that IMP become the stander of treatment for long bone fractures, success and easy, commonly used, and less traumatic to ill animal. The good results of using FGSR for internal fixation which achieved radiographically by callus formation due to periosteal reaction around the fracture line, during healing process that conducted with [17].

Easy to use with low cost, less of bone exposed comparing with other methods (bone plate), Minimal amount of tissue damage and distraction. Do not need to support it with the external fixation, Do not lead to deformity of loss the function of the affected limbs or any joint stiffness $[8,14]$. The FGSR 


\section{Open Access Journal of Veterinary Science \& Research}

not change during sterilization or exposed to different type and dosage of irradiation, as proved practically [8] because of its element mixtures. The Inflammatory signs which occurs immediately after the surgical operation during the inflammatory stages and the clinical observation, they returned to normal condition in few days after operation, all these signs were normal during the inflammatory phase post operation as mentioned by $[6-10,18]$.

The surgical incision healed normally without complication except some cases suffered from stitch abscess, which also healed rapidly after local treatment, and this information agree with [19], and the affected limb began to use the limbs in the movement and support the weight several days p. o., this statement agree with [19] that lameness disappear gradually especially in stable fracture, and the inflammatory signs and healing process of the wound are normally occurs after surgical operation in rabbits. No- body rejection or complication during the healing processing.

The FGSR can be easy and successfully used for internal fixation of induced transverse fractures in the mid shift of the femoral bone besides it can fix and implanted the natural xeno-bony implantation of the sheep and calves origin and the synthetic nano-bony implantation [6-11]. The intramedullary pins remain in the place during all healing periods with now signs of infection, and the animal comfortable with the device, and this agree with [20], who refer that the intramedullary rod generally remains in the bone for the life of the patient can be removed if it causes pain or other problem

\section{$>$ Disadvantages:}

The animals suffering from pain when insert the pin for fracture fixation, or when remove the pins it need other operation for that [20].Not lead to immobilization, Not stopped the circulation movement, Lead to bone marrow destruction and the inner blood circulation and may lead to infection due to iterance of microorganism, May lead to osteomylitis, May lead to loss in the pins, Less stable fixation, Slower return to function, Secondary bone union, More involved after care $[8,14,15]$.

\section{Radiological Findings}

\section{$>$ Advantages:}

Fracture healing by secondary bone repair (endochondral ossification), with new hard callus formation continued by remodeling phase as in the IMP $[4,5]$. Excessive amount of the new bone formation around the fracture site because the micro movement of the intramedullary pin, with a gap between the two femoral fragments. The healing processing by secondary fracture healing, in that the periosteal reaction and callus formation which later converted to mature or hard callus which increase in volume and density and gradually pass the fracture gap to form the bony bridge, to join the two fractures fragment, with disappear of the fracture line from the proximal and distal part of the bone away from the fracture line, the cortex still not completely incorporated within the fracture line and this agree with [17].

All the radiographic finding of FGSR during fracture healing processing is the same compare with medical IMP as published by $[8,9,21]$. Which started from periosteial reaction with gradually disappear the fracture line and gap. The remodeling phase, which achieved by homogenous external callus with smooth outer border and stop formation of more callus around the fracture line and the cortex begins to incorporated and this agree with $[17,22]$, patency of the medullary canal was maintained due to the presence of the intra nails $[17,24,25]$. Complete healing fracture process occurs from the signs of radiological union, hard callus around the fracture produced the bridge and completely disappear the fracture line $[6,9,24,25]$.

\section{$>$ Disadvantages:}

The FGSR fill bony canal and interfere with bone marrow and circulation $[14,15]$.

\section{Conclusion}

* The advantages of using FGSR can be summarized by, The FGSR is suitable methods for internal fixation of the induced femoral fracture in rabbits because it easy to use, easy to modified, popular, not expensive easy to find, with no complication or body rejection

* The healing processing and remodeling phase during using FGSR is by secondary bone union and the FGSR can support the body to bear the weight and give proper alignment to fractures fragments.

- All the chemical and physical analysis of FGSR is the same as in the medical IMP, and not change during different methods of sterilization, not irritant, not corrosive not carcinogenic and not altered during different types of irradiation or electromagnetic field.

* The disadvantages are very rare or absent which include, Pain, infection, joint trauma, with failed in bone alignment. Lateral deviation of the stifle joint with improper limb gait, fracture complication with comminuted or multible re-fracture may occur. With Some very little case shows the pin going out the body or it not fit to support weight or fracture alignment and some of the FGSR mineral content is not standard as in the medical IMP, and Removing the intramedullary pinning after fracture repair and radiological union need other operation. 


\section{Open Access Journal of Veterinary Science \& Research}

\section{Acknowledgment}

Special thanks for all whom sharing to support this study or give thought and decision to complete the research requirements.

\section{References}

1. Brinker WO, Piermatti DL, Flo GL (1991) Handbook of small animal orthopaedics and fracture treatment. Printed in the United states of America, W.B. Saunders Company, Phiadilphia London, USA, pp: 72-79.

2. Archibald J (1974) Canine Surgery, $2^{\text {nd }}$ (Edn.), American Veterinary Publication, INC., California.

3. Marshall T, Browner BD (2012) Chapter20: Emergency care of musculoskeletal injuries. In: Courtney $\mathrm{M}$, Townsend Jr, Sabiston textbook of surgery: the biological basis of modern surgical practice, Elsevier, pp: 480-520.

4. Christodoulou A, Terzidis Ploumis A, Metsovitisi S, Koukoulidiis A, Toptsis C (2005) Supracondylar femoral fractures in elderly patients treated with the dynamic condylar serew and the retrograde intramedullary nail: a comparative study of the two methods. Arch orthop Trauma Surg 125(2): 73-79.

5. Krettek C, Miclau T, B lauth M, Lindsey RW, Donow C, et al. (1997) J Bone Joint Surg IBr 79-B: 4-8.

6. Nazht Humam H, Raffal A Omar, Hatem K Ahmed (2018) Sheep rib xenograft femoral bone implant in rabbits. Journal of Veterinary Research 22(4): 290-299.

7. Nazht, Humam H, Raffal A Omar, Muna RA AlDahhan, Hatem kAhmed (2018) Effect of Low Level Laser Therapy on the Sheep Ribs Xeno Graft in the Treatment of Rabbits Long Bone Fractures. The Ninth International Scientific Academic Conference Under the Title "Contemporary trends in social, human, and natural sciences", pp: 100106.

8. Nazht Humam H (2019) Using Food Grate Stainless Steel Rods for Internal Fixation of Transverse Fractures in Rabbits. Vet Sci Res 4(3): 000188.

9. Nazht Humam H, Rania K, Sumay M (2019) Radiological Evaluation of Fractures Healing Processing Fixed by Food Grate Stainless Steel Rods in Rabbits. Vet Sci Res 4(3): 000189.

10. Nazht Humam H, Raffal A Omar, Muna R A Al Dahhan, Rania Khedr Khaleef, Sumaya Mohammed Kareem, et al. (2019) Estimation of Alkaline Phosphatase Enzymes Level in the Femoral Transverse Fractures Healing in
Rabbits. The 10th International Scientific Conference. Under the Title "Geophysical, Social, Human and Natural Challenges in a Changing Environment", pp: 97-107.

11. Nazht Humam H, Ammar MH, Almahmoud Dhyaa Ab, Abood (2019) Using Xeno -Bovine Bony Implantation as Space Filler in Femoral Defect in Rabbits 5Contemporary International Scientific Forum for Educational, Social, Human, Administrative and Natural Sciences "Present Vs Future, pp: 244-252.

12. Leung, Kwok-Sui, Kempf Ivan, Alt Volker, Taglang Gilbert, Haarman HJTM, et al. (2006) Practice of intramedullary locked nails: new developments in techniques and applications. Birkhäuser, pp: 100.

13. Jonathan Cluett (2018) Medically reviewed by a boardcertified physician.

14. Frik EJ, Winer RE, Mosier JE (1948) Treatment of fractures by intramedullary pining. North Am Vet 29(2): 95-97.

15. Slatter Douglas H (1985) Small Animal surgery.

16. Abd-Alreda Ahmed Mohammad (2016) Influence of ketorolac on bone repair in rabbits. M.Sc. thesis, Veterinary medicine college, Baghdad University.

17. Teot L (1987) L'enclouage centromedullaire elastique stable chez l'enfant. Cahier d'enseignement de la SOFCOT no 28 Expansion scientific que francaise, Paris, pp: 71-90.

18. Lastayo PC, Winters KM, Hardy M (2003) Fracture healing: bone healing, fracture management, and current concepts related to the hand. J Hand Ther 16(2): 81-93.

19. Singh SG, Kumar S, Matti SK (2000) Reconstruction of ulnar segmental defects with autograft and ceramic biomaterials: A Scanning Electron Microscope (SEM) study. Indian J Anim Sci 70: 276-278.

20. Stiftler Ks (2004) Internal fracture fixation. Clin Tech Small Anim Pract 19(3): 105-113.

21. Winquist RA, Hansen ST (1980) Comminuted fractures of the femoral shaft treated by Intramedullary nailing. Orthop Clin North Am 11(3): 633-648.

22. Lascombes $P$ (2009) Flexible intramedullary nailing in Children, $1^{\text {st }}$ (Edn.), Lascombes.

23. Ruedi TP, Buckley RE, Moran CG (2007) AO Principles of Fracture Management.

24. Thelen M, Ritter G, Bucheler E (1993) Radiologische 


\section{Open Access Journal of Veterinary Science \& Research}

Diagnostik der Verletzungen von knochen und Gelenken. Ein leitfaden fur lologen und Traumatologen. Thieme.
25. McRae R, Esser M (2008) Practical fracture Treatment. Churchill Livingstone. 